Supplement of Biogeosciences, 17, 581-595, 2020

https://doi.org/10.5194/bg-17-581-2020-supplement

(c) Author(s) 2020. This work is distributed under

the Creative Commons Attribution 4.0 License.

(c) (1)

Supplement of

\title{
Dissolved organic carbon mobilized from organic horizons of mature and harvested black spruce plots in a mesic boreal region
}

Keri L. Bowering et al.

Correspondence to: Keri L. Bowering (klbowering@mun.ca)

The copyright of individual parts of the supplement might differ from the CC BY 4.0 License. 
Table S1. Results of repeated measure linear mixed models assessing the effects of plot type. collection day and the interactive effect of collection day and plot type (forest and harvested) on temporal variations in lysimeter captured dissolved organic carbon (DOC) fluxes, water fluxes and DOC concentration. P-values shown with significant results in bold (alpha $=0.05)$. Post hoc least squares means tests used to determine significant differences between plot type shown in Figure 1D-F (asterisks)

\begin{tabular}{|c|c|c|c|}
\hline & df & F value & $\mathrm{p}$-value \\
\hline \multicolumn{4}{|l|}{ 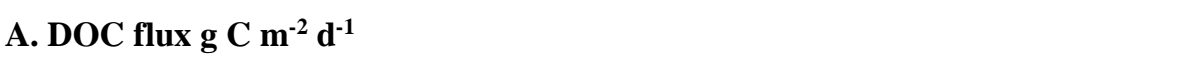 } \\
\hline plot type & 1 & 3.88 & 0.0616 \\
\hline collection day & 29 & 23.49 & $<0.0001$ \\
\hline plot type $\mathrm{x}$ collection day & 29 & 1.51 & 0.0183 \\
\hline \multicolumn{4}{|l|}{ B. Water flux $L \mathbf{m}^{-2} \mathrm{~d}-1$} \\
\hline plot type & 1 & 4.98 & 0.0361 \\
\hline collection day & 29 & 26.71 & $<0.0001$ \\
\hline plot type $\mathrm{x}$ collection day & 29 & 2.36 & 0.0004 \\
\hline \multicolumn{4}{|l|}{ C. $[D O C] \mathrm{mg} \mathrm{L}^{-1}$} \\
\hline plot type & 1 & 7.27 & 0.0132 \\
\hline collection day & 24 & 48.45 & $<0.0001$ \\
\hline plot type $\mathrm{x}$ collection day & 24 & 2.90 & $<0.0001$ \\
\hline
\end{tabular}


Table S2. Results of one way plot nested ANOVAs assessing the effects of plot type on annual lysimeter captured dissolved organic carbon (DOC) fluxes, water fluxes and DOC concentration. P-values shown with significant results in bold $($ alpha $=0.05)$. Plot type differences shown in Figure 2A-C (asterisks).

df F value p-value

A. DOC flux $\mathrm{g} \mathrm{C} \mathrm{m}^{-2}$ plot type

$1 \quad 23.49 \quad \mathbf{0 . 0 0 8 4}$

B. Water flux $\mathrm{L} \mathrm{m}^{-2}$

plot type

$\begin{array}{lll}1 & 10.07 & \mathbf{0 . 0 3 3 7}\end{array}$

C. $[D O C] \mathrm{mg} \mathrm{L}^{-1}$

$\begin{array}{lll}1 & 7.27 & 0.0903\end{array}$


Table S3. Linear mixed effects model results examining the effects of plot type, sample year $(2013,2014,2015)$, and their interaction on soil respiration. $\alpha=0.05$

\begin{tabular}{llll}
\hline Source & DF & F & p-value \\
\hline Plot type & 1 & 4.79 & 0.0721 \\
Year & 2 & 87.28 & $<\mathbf{0 . 0 0 0 1}$ \\
Year x Plot type & 2 & 5.13 & $\mathbf{0 . 0 0 6 0}$ \\
& & & \\
\hline
\end{tabular}


Table S4. Least square means for multiple comparisons of soil respiration $\left(\mathrm{CO}_{2} \mathrm{~m}^{-2} \mathrm{~s}^{-1}\right)$ in black spruce forests across plot type (harvested and forest) and sample years (2013-2015). Soil respiration was measured during the snow-free growing season. $\alpha=0.05$

\begin{tabular}{lllllll}
\hline Treatment_Year & Mean & Std. & DF & t & p-value \\
& & Diff. & Error & & & \\
\hline Forest_2013 & Harvested_2013 & -0.465 & 1.488 & 2148 & -0.31 & 0.7546 \\
& Forest_2014 & -7.227 & 0.898 & 2148 & -8.05 & $<.0001$ \\
& Forest_2015 & -8.562 & 0.922 & 2148 & -9.28 & $<.0001$ \\
Harvested_2013 & Harvested_2014 & -1.337 & 0.898 & 2148 & -1.49 & 0.1366 \\
& Harvested_2015 & -3.741 & 0.922 & 2148 & -4.06 & $<.0001$ \\
Forest_2014 & Harvested_2014 & 5.424 & 1.524 & 2148 & 3.56 & $\mathbf{0 . 0 0 0 4}$ \\
& Forest_2015 & -1.334 & 0.953 & 2148 & -1.40 & 0.1619 \\
Harvested_2014 & Harvested_2015 & -2.403 & 0.953 & 2148 & -2.52 & $\mathbf{0 . 0 1 1 8}$ \\
Forest_2015 & Harvested_2015 & 4.355 & 1.552 & 2148 & 2.81 & $\mathbf{0 . 0 0 5 1}$ \\
\hline
\end{tabular}


Table S5. Mean cumulative soil respiration $\left(\mathrm{R}_{\mathrm{s}}\right)$

for the snow-free growing season in 4 harvested plots and 4 forest plots. $\mathrm{N}$ refers to number of plot scale replicates. Values connected by the same letter are not significantly different determined by lsmeans tests shown in table S4 .

\begin{tabular}{llll}
\hline Treatment & Year & $\mathbf{N}$ & $\begin{array}{l}\mathbf{R}_{\mathbf{s}} \\
\left.(\mathrm{Mg} \mathrm{C} \mathrm{ha})^{1}\right)\end{array}$ \\
\hline Forest & $\mathbf{2 0 1 3}$ & 4 & $6.06 \pm 0.41^{\mathrm{a}}$ \\
& $\mathbf{2 0 1 4}$ & 4 & $7.14 \pm 0.55^{\mathrm{bc}}$ \\
& $\mathbf{2 0 1 5}$ & 4 & $7.85 \pm 0.59^{\mathrm{c}}$ \\
Harvested & $\mathbf{2 0 1 3}$ & 4 & $5.53 \pm 0.32^{\mathrm{a}}$ \\
& $\mathbf{2 0 1 4}$ & 4 & $6.20 \pm 0.27^{\mathrm{a}}$ \\
& $\mathbf{2 0 1 5}$ & 4 & $6.49 \pm 0.29^{\mathrm{b}}$ \\
& & & \\
\hline
\end{tabular}


Table S6. Regression analysis among soil respiration, $\mathrm{R}_{\mathrm{s}}\left(\mu \mathrm{mol} \mathrm{CO} \mathrm{CO}^{-2} \mathrm{~s}^{-1}\right)$, soil temperature, $\mathrm{T}_{\mathrm{s}}\left({ }^{\circ} \mathrm{C}\right)$, and soil moisture content, $\mathrm{M}_{\mathrm{s}}\left(\mathrm{m}^{3} / \mathrm{m}^{3}\right)$, and $\mathrm{T}_{\mathrm{s}} \times \mathrm{M}_{\mathrm{s}}$ interactions in the harvested and forest plots. $\mathrm{R}_{\mathrm{s}}, \mathrm{T}_{\mathrm{s}}$, and $\mathrm{WC}_{\mathrm{s}}$ were measured concurrently at biweekly interval during the snow free season (May 1Nov 30) from 2013-2015. $\alpha=0.05$

\begin{tabular}{lllllll}
\hline Plot type & Variable & DF & $\begin{array}{l}\text { Parameter } \\
\text { Estimate }\end{array}$ & $\begin{array}{l}\text { Standard } \\
\text { Error }\end{array}$ & $\mathbf{t}$ & p-value \\
\hline Forest & Intercept & 1 & 0.986 & 0.579 & 1.7 & 0.0892 \\
& $\mathrm{~T}_{\mathrm{s}}$ & 1 & 0.278 & 0.055 & 5.08 & $<.0001$ \\
& $\mathrm{M}_{\mathrm{s}}$ & 1 & -0.037 & 0.019 & -1.93 & $\mathbf{0 . 0 5 4 4}$ \\
& $\mathrm{T}_{\mathrm{s}} \times \mathrm{M}_{\mathrm{s}}$ & 1 & 0.004 & 0.002 & 2.17 & $\mathbf{0 . 0 3 0 6}$ \\
& & & & & & \\
Harvested & Intercept & 1 & 1.543 & 0.432 & 3.57 & 0.0004 \\
& $\mathrm{~T}_{\mathrm{s}}$ & 1 & 0.145 & 0.032 & 4.60 & $<.0001$ \\
& $\mathrm{M}_{\mathrm{s}}$ & 1 & -0.071 & 0.014 & -4.99 & $<.0001$ \\
& $\mathrm{~T}_{\mathrm{s}} \mathrm{x} \mathrm{M}_{\mathrm{s}}$ & 1 & 0.006 & 0.001 & 5.58 & $<.0001$ \\
\hline
\end{tabular}


Table S7. Calibration equations for field measured soil water content (WC) at $5 \mathrm{~cm}$ depth in Forest and Harvested plots. Corrected WC derived from infiltration and evaporation experiments at residual and matrix saturation (see Table 4).

Field Corrected Calibration Equation

WC $(\mathrm{x}) \quad \mathrm{WC}(\mathrm{y})$

\begin{tabular}{rlll}
\hline \multicolumn{1}{l}{ Forest } & & & \\
Residual & 0.04 & 0.18 & $\mathrm{y}=1.5769 \mathrm{x}+0.1219$ \\
Matrix & 0.17 & 0.39 &
\end{tabular}

\section{Harvested}

$\begin{array}{rrrr}\text { Residual } & 0.10 & 0.20 & \mathrm{y}=2.0 \mathrm{x} \\ \text { Matrix } & 0.23 & 0.46 & \end{array}$

